\title{
Managing Clinical Trials for Alzheimer's Disease During the COVID-19 Crisis: Experience at Fundació ACE in Barcelona, Spain
}

\author{
Carla Abdelnour ${ }^{\mathrm{a}, \mathrm{b}, 2}$, Ester Esteban de Antonio ${ }^{\mathrm{a}, 2}$, Alba Pérez-Cordón ${ }^{\mathrm{a}}$, Asunción Lafuente ${ }^{\mathrm{a}}$, \\ Mar Buendía ${ }^{a}$, Ana Pancho ${ }^{a}$, Sara Jofresa ${ }^{a}$, Nuria Aguilera ${ }^{a}$, Marta Ibarria $^{a}$, Rosario Cuevas ${ }^{\mathrm{a}}$, \\ Laia Cañada $^{\mathrm{a}}$, Anna Calvet ${ }^{\mathrm{a}}$, Susana Diego ${ }^{\mathrm{a}}$, Antonio González-Pérez ${ }^{\mathrm{a}}$, Adela Orellana ${ }^{\mathrm{a}, \mathrm{b}}$, \\ Laura Montrreal $^{\mathrm{a}}$, Laura de Jorge ${ }^{\mathrm{c}}$, Marta Marquiéa,b ${ }^{\mathrm{a}}$, Alba Benaque ${ }^{\mathrm{a}}$, Miren Gurruchaga ${ }^{\mathrm{a}}$, \\ Lluís Tárraga ${ }^{\mathrm{a}, \mathrm{b}}$, Agustín Ruiz ${ }^{\mathrm{a}, \mathrm{b}}$ and Mercè Boada ${ }^{\mathrm{a}, \mathrm{b}, *}$ for the Research Center and Memory Clinic, \\ Fundació ACE ${ }^{1}$ \\ ${ }^{a}$ Research Center and Memory Clinic, Fundació ACE, Institut Català de Neurociències Aplicades, Universitat \\ Internacional de Catalunya, Barcelona, Spain \\ ${ }^{\mathrm{b}}$ Networking Research Center on Neurodegenerative Diseases (CIBERNED), Instituto de Salud Carlos III, Spain \\ ${ }^{\mathrm{c}}$ SYNLAB Diagnóstico Globales, SA
}

Accepted 8 September 2020

\begin{abstract}
.
Background: The COVID-19 pandemic has brought great disruption to health systems worldwide. This affected ongoing clinical research, particularly among those most vulnerable to the pandemic, like dementia patients. Fundació ACE is a research center and memory clinic based in Barcelona, Spain, one of the hardest-hit countries.

Objective: To describe the ad-hoc strategic plan developed to cope with this crisis and to share its outcomes.

Methods: We describe participants' clinical and demographic features. Additionally, we explain our strategic plan aimed at minimizing the impact on clinical trial research activities, which included SARS-CoV-2 RT-PCR and IgG serological tests to all participants and personnel. The outcomes of the plan are described in terms of observed safety events and drop-outs during the study period.

Results: A total of 130 patients were participating in 16 active clinical trials in Fundació ACE when the lockdown was established. During the confinement, we performed 1018 calls to the participants, which led to identify adverse events in 26 and COVID-19 symptoms in 6. A total of 83 patients (64\%) could restart on-site visits as early as May 11, 2020. All SARS-CoV-2 RT-PCR diagnostic tests performed before on-site visits were negative and only three IgG serological tests were positive. Throughout the study period, we only observed one drop-out, due to an adverse event unrelated to COVID-19.
\end{abstract}

\footnotetext{
${ }^{*}$ Correspondence to: Dr. Mercé Boada, Research Center and Memory Clinic, Fundació ACE, Institut Català de Neurociències Aplicades, Gran Via de Carles III, 85 BIS, 08028 Barcelona, Spain. Tel.: +34 9343047 20; E-mail: mboada@fundacioace.org.

${ }^{1}$ For the Research Center and Memory Clinic, Fundacio ACE: Isabel Hernández ${ }^{\mathrm{a}, \mathrm{b}}$, Montserrat Alegret $^{\mathrm{a}, \mathrm{b}}$, Pilar Cañabate ${ }^{\mathrm{a}, \mathrm{b}}$, Isabel Rodríguez ${ }^{\mathrm{a}, \mathrm{b}}$, Maitee Rosende-Roca ${ }^{\mathrm{a}}$, Juan Pablo Tartari ${ }^{\mathrm{a}}$,
}

\footnotetext{
Rogelio López ${ }^{\mathrm{a}}$, Silvia Gil ${ }^{\mathrm{a}}$, Liliana $\operatorname{Vargas}^{\mathrm{a}}$, Ana Mauleón ${ }^{\mathrm{a}}$, Ana Espinosa ${ }^{a, b}$, Gemma Ortega ${ }^{a, b}$, Angela Sanabria ${ }^{a, b}$, Emilio Alarcón $^{\mathrm{a}}$, Mariola Moreno ${ }^{\mathrm{a}}$, Silvia Preckler ${ }^{\mathrm{a}}$, Natalia Roberto ${ }^{\mathrm{a}}$, Sergi Valero $^{\mathrm{a}}$, Itziar de Rojas ${ }^{\mathrm{a}}$, Sonia Moreno-Grau ${ }^{\mathrm{a}, \mathrm{b}}$, and Elvira Martín ${ }^{\mathrm{a}}$

${ }^{2}$ These authors contributed equally to this work.
} 
Discussion: The plan implemented by Fundació ACE was able to preserve safety and integrity of ongoing clinical trials. We must use the lessons learned from the pandemic and design crisis-proof protocols for clinical trials.

Keywords: Alzheimer's disease, clinical trials, coronavirus, pandemics, telemedicine

\section{INTRODUCTION}

In December 2019, an outbreak of an atypical pneumonia of unknown origin was identified in Wuhan, China. The new coronavirus responsible for the disease, severe acute respiratory syndrome coronavirus-2 (SARS-CoV-2), is characterized by rapid human-to-human transmission [1]. Consequently, the outbreak of Coronavirus Disease 2019 (COVID-19) has rapidly spread globally and eventually declared a pandemic by the World Health Organization on March 11, 2020 [2]. It entails a global health crisis by its own consequences and by the impact of the measures that have been taken in order to control the outbreak, affecting all areas of care and research.

As a result, in relation to clinical trials, Regulatory Agencies (Food and Drug Administration, European Medicines Agency, and Spanish Agency for Medicines and Health Products) published guidance for industry, investigators, and institutional review boards (IRBs) on conduct of clinical trials of medical products during the COVID-19 pandemic [3-5], since challenges may arise from quarantines, site closures, travel limitations, interruptions to the supply chain for the investigational product (IP), or even infection of site personnel or trial subjects. The key issue of all these recommendations is the safety of clinical trials participants. Firstly, they provided general considerations to assist sponsors in assuring the safety of trial participants, maintaining compliance with good clinical practice and minimizing risks to trial integrity, recognizing that protocol modifications, as well as protocol deviations, may be required to deal with it [3]. Such modifications should be documented properly and, although changes to the protocol to protect the life and well-being of research participants may be implemented without IRB approval, they are required to be reported afterwards [3-5]. Secondly, sponsors, in consultation with clinical investigators and IRBs/Independent Ethics Committees (IECs), may determine the continuation or discontinuation of the administration of the IP or even the participation in the trial, to protect participant's safety, welfare, and rights, considering each individual circumstance. Thirdly, it is advisable to implement alternative and effective methods for safety monitoring (for instance, by phone contacts, virtual visits, alternative location visits). It is also important to keep trial participants informed of changes to the study and monitoring plans. Fourthly, the importance to capture specific information in the case report form that explains the basis of possible missing data due to COVID-19 is also highlighted. Finally, regarding IP's distribution, alternative secure delivery methods may be an option for some selfadministered IPs (carried out by Pharmacy Services or authorized courier services) or alternative administration methods (for instance, home nursing) could be considered, to prevent avoidable visits to sites and to provide the trial participants with needed treatments [3-5]. The European (EMA) and Spanish (AEMPS) Agencies addressed some specific measures $[4,5]$ regarding Informed Consent, that can be obtained verbally (by telephone or video conference), but it is necessary to document it in the clinical history and to confirm it afterwards in writing, with patient and investigator signatures, being able to use digital media to do so. Additionally, remote monitorization of source data is recommended.

In response to this situation, some clinical and research centers have developed adaptation measures [6-8], but there is scarce literature about it. For example, most of oncology trials are ongoing in an institutional setup where preemptive measures to contain the virus are already instituted [9]. A Chinese center reported that, despite numerous protocol deviations, outcomes and questionnaires could be collected remotely and oral drugs were delivered under careful management [10].

It is important to note that the disruption of important clinical research by the COVID-19 pandemic is linked also to the particular vulnerability of older adults, to whom the impact of the pandemic is greatest [11] and are the main target subjects of Alzheimer's disease (AD) clinical trials. Taking this into account, every effort should be made to preserve the integrity and value of critical ongoing research in $A D$ while balancing the dynamic risks associated with study participation during an outbreak [12]. Otherwise abrupt interruption of these research studies could have long lasting consequences for the field of $\mathrm{AD}$ research [13]. 
Fundació ACE is a private non-profit organization providing care based on a holistic and multidisciplinary model to patients with cognitive disorders and their families, for 25 years in Barcelona, Spain [14]. To mitigate the disruption of $\mathrm{AD}$ clinical trials due to COVID-19, new interventions need to be implemented and therefore, being Fundació ACE's model dynamic by nature [15], a contingency plan was put in place according to the events to better accommodate patients in this new scenario ensuring continuity of clinical trials.

The aim of this article is to describe the Fundació ACE Clinical Trials Strategic Plan developed to cope with COVID-19 outbreak and summarize its outcomes.

\section{METHODS}

Following news on how the outbreak in northern Italy was leading to a collapsed health system, by early March it became clear that the advent of the outbreak to neighboring countries was imminent. At this point, a contingency plan was designed to prepare for an anticipated suspension of all face-to-face activities at Fundació ACE including the day care center, the memory clinic, and the research and clinical trials units. The plan defined the steps to follow for a rapid transition from an in-person to a virtual model of care. Recruitment of new clinical trial participants was stopped at this stage and meetings with sponsors were held to determine the actions to be taken in the different possible scenarios. The declaration of the state of alarm by the Spanish government on March 14, 2020 precipitated the events. The severe restrictions imposed for all non-essential activities and mobility (ex. safe passages required) lead to the suspension of all treatments and in-person visits, activating the contingency plan. Thus, weekly calls (either phone or videoconference calls) to all study participants were gradually established and a short questionnaire was performed during these calls to collect relevant information regarding the patient's social situation, presence of COVID-19 symptoms, adverse events, and changes in concomitant medication (see Supplementary Material 1).

The sponsors made changes to the study protocols that allowed performing some of the due neurological and neuropsychological assessments (behavioral and functional scales) remotely by phone call of videoconference (see Supplementary Material 2). For this purpose, we have received specific guidelines and training. No cognitive assessment was permitted to be conducted remotely (Mini-Mental State Examination [MMSE], Alzheimer's Disease Assessment Scale-Cognitive Subscale, Repeatable Battery for the Assessment of Neuropsychological Status, and Free and Cued Selective Reminding Test).

A specific protocol to manage trial adverse events and suspected cases of COVID-19 was established. Given the limited availability of SARS-CoV-2 testing, home nursing drug administration was discarded to preserve safety of professionals, participants, and caregivers. Finally, depending on status and characteristics of each trial, virtual follow-up visits and courier service delivery of oral study medication were adopted when possible in agreement with sponsors.

Almost simultaneously, we started preparing for future face-to-face visits. We arranged SARS-CoV-2 RT-PCR testing for all personnel and study participants with an external diagnostic laboratory. Staff was split into two teams to minimize exposure and cross-contamination among healthcare professionals. Safety protocols were adapted to include the use of personal protective equipment (PPE), and to ensure that physical distance was maintained during visits by using physical barriers and restricting mobility. Thus, all personnel (including administrative staff) had to wear PPE consisting of scrub suits, shoe covers, and face masks. Those in direct contact with patients (study nurses, subinvestigators, and raters) had to wear FFP2 masks, goggles, or face shields, along with surgical gowns and gloves. Training sessions were held to explain the correct use of PPE. Patients and caregivers were also provided with face masks, shoe covers, and a sealed bag for their personal items. Patients were also provided with gowns. Brain MRIs and PET imaging were performed in external facilities, away from hospitals where COVID-19 patients are seen.

On May 4, 2020, SARS-CoV-2 RT-PCR testing of the clinical trials unit personal and study participants started as well as MRI imaging. On May 11, 2020, on-site study drug administration and on-site visits began for a selected group of participants who fulfilled the following prioritization criteria: 1) subjects who should receive the IP per calendar and who would otherwise miss the corresponding dose if the visit was not performed, 2) participants with longest time without a safety MRI, and 3) those running out of oral medication. Additionally, we took into consideration the need to keep scheduled visits as unchanged as possible. 


\section{Descriptive statistics}

We provide a detailed description of patients being screened or those randomized in clinical trials conducted at Fundació ACE when COVID-19 outbreak occurred in Spain. Thus, based on data from the last available assessment, we describe the study population in terms of age, sex, years of education, MMSE score, Clinical Dementia Rating (CDR), etiological diagnoses, comorbidities (with special emphasis on cardiovascular conditions reported to be COVID-19 risk factors), type of study drug, and study phase. Categorial variables were described by using percentages, whereas mean and standard deviations were used to summarize quantitative variables.

The study period includes twelve weeks from the suspension of face-to-face activity, on March 16, until June 5, 2020. During this period, we identified the presence of COVID-19 symptoms on patients or relatives using a short questionnaire designed ad-hoc. We ascertained adverse events and changes in concomitant medication during this period. We also describe the results of SARS-CoV-2 testing (both RT-PCR and IgG serology tests) among the subset of participants that initiated on-site visits during the study period. We compared the proportions of patients and professionals with positive IgG serological tests with estimates reported for the province of Barcelona [16] using Fisher exact tests in Stata (Version 12, College Station, TX).

\section{SARS-CoV-2 RT-PCR testing}

RT-PCR SARS-CoV-2 testing was conducted by an external laboratory (SYNLAB SL) on samples obtained from nasopharyngeal and oropharyngeal swabs using VIASURE SARS-CoV-2 S gene Real Time PCR Detection Kit (CerTest; Prolabs). Briefly, RNA was extracted from the samples to obtain the complementary DNA (cDNA) with a reverse transcription process (RT). Then, cDNA is amplified with SARS-CoV-2-specific probes. After the amplification, SARS-CoV-2 is detected on channel $475 / 520$ with and internal control detected on channel $530 / 565$.

\section{Determination of anti SARS-CoV-2 Ig G}

To study the presence of $\operatorname{IgG}$ antibodies for SARS-CoV-2 our laboratory performed the following analysis: all sera plasma was obtained by centrifu- gation of blood collected from patients in tubes BD Vacutainer SSTII Advance $\left(2000 \times \mathrm{g} 10 \mathrm{~min},+4^{\circ} \mathrm{C}\right)$. Anti SARS-CoV-2 Ig G from serum samples was determined in a semi quantitative manner using ELISA SARS-Coronavirus-2 (SARS-CoV-2) IgG kits of Euroimmun (EI 2606-9601, Germany). Samples were run by duplicate. The assay was performed according manufacturer's protocol. Reagents wells are coated with recombinant structural protein (S1 domain) of SARS-CoV-2. The optical density (OD) was detected at $450 \mathrm{~nm}$, and a ratio of the reading of each sample to the reading of the calibrator, included in the kit, was calculated for each sample (OD ratio). OD ratio $<0.8$ was considered negative and OD ratio $\geq 1.1$ considered positive. OD ratio between 0.8 and 1.1 was considered uncertain. The kit also included a positive and a negative control samples.

\section{RESULTS}

There were 16 active clinical trials in Fundació ACE (Barcelona) at the time the state of emergency was declared in Spain and all non-essential activities were suspended on March 14, 2020. In three of these trials, the recruitment period was open. In the previous two months (January and February 2020), we had recruited 16 and 23 new patients respectively. At this point, a total of 130 individuals were either undergoing screening $(n=22,17 \%)$ or had already been randomized in one of these trials $(n=108,83 \%)$. As shown in Table 1, most patients corresponded to trials of anti-amyloid (52\%), or anti-tau (32\%) therapies. Among randomized patients, most were enrolled in trials that involved either intravenous (IV; $52 \%$ ) or subcutaneous (29\%) administration of IP. Only $19 \%$ of them were receiving an oral drug.

The characteristics of trial participants are described in Table 2. According to the most recent CDR assessment, most patients had a CDR score of $0.5(n=52,40 \%)$ or $1(n=61,47 \%)$. Only 17 patients $(13 \%)$ had a CDR score of 2 or more. Mean age was 73.8 ( $\mathrm{SD}=6.6)$, with $92 \%$ of them being 65 years or older. Most clinical trial participants were females $(n=80,62 \%)$ and had completed on average 9 years of education. Mean MMSE score was 21.7 $(\mathrm{SD}=4.9)$, ranging from $25.1(\mathrm{SD}=2.2)$ among those with CDR of 0.5, to 14.0 ( $\mathrm{SD}=5.4$ ) for patients with CDR above 1 . The most frequent comorbidity was hypertension $(46 \%)$, followed by diabetes mellitus (12\%), and thyroid disease (7\%). 
Table 1

Clinical trial participants by study phase and type of treatment and route of administration

\begin{tabular}{lcccccc}
\hline & $\begin{array}{c}\text { Screening } \\
\text { phase } \\
(n=22)\end{array}$ & $\begin{array}{c}\text { Randomized } \\
(n=108)\end{array}$ & $\begin{array}{c}\text { Total } \\
(n=130)\end{array}$ \\
\hline Investigational product & $n$ & $\%$ & $n$ & $\%$ & $n$ & $\%$ \\
$\quad$ Anti-tau (4 trials) & 2 & $9 \%$ & 39 & $36 \%$ & 41 & $32 \%$ \\
$\quad$ Anti-amyloid (6 trials) & 12 & $55 \%$ & 55 & $51 \%$ & 67 & $52 \%$ \\
$\quad$ Neuroprotection (1 trial) & 8 & $36 \%$ & 3 & $3 \%$ & 11 & $8 \%$ \\
$\quad$ Anti-neuroinflammation (1 trial) & 0 & $0 \%$ & 1 & $1 \%$ & 1 & $1 \%$ \\
$\quad$ Cognitive enhancer (1 trial) & 0 & $0 \%$ & 6 & $6 \%$ & 6 & $5 \%$ \\
$\quad$ Cognitive behavioral therapy (3 trials) & 0 & $0 \%$ & 4 & $4 \%$ & 4 & $3 \%$ \\
Route of administration & & & & & & \\
$\quad$ Oral & 15 & $68 \%$ & 21 & $19 \%$ & 36 & $28 \%$ \\
$\quad$ Intravenous & 7 & $32 \%$ & 56 & $52 \%$ & 63 & $48 \%$ \\
$\quad$ Subcutaneous & 0 & $0 \%$ & 31 & $29 \%$ & 31 & $24 \%$ \\
\hline
\end{tabular}

Table 2

Clinical trial participants' characteristics and events by CDR status

\begin{tabular}{|c|c|c|c|c|c|c|c|c|}
\hline \multirow[b]{2}{*}{ Sex } & \multicolumn{2}{|c|}{$\mathrm{CDR}=0.5(n=52)$} & \multicolumn{2}{|c|}{$\mathrm{CDR}=1(n=61)$} & \multicolumn{2}{|c|}{$\mathrm{CDR} \geq 2(n=17)$} & \multicolumn{2}{|c|}{ Total $(n=130)$} \\
\hline & $n$ & $\%$ & $n$ & $\%$ & $n$ & $\%$ & $n$ & $\%$ \\
\hline Male & 23 & $44 \%$ & 23 & $38 \%$ & 4 & $24 \%$ & 50 & $38 \%$ \\
\hline Female & 29 & $56 \%$ & 38 & $62 \%$ & 13 & $76 \%$ & 80 & $62 \%$ \\
\hline \multicolumn{9}{|l|}{ Age } \\
\hline $55-64$ y & 3 & $6 \%$ & 6 & $10 \%$ & 2 & $12 \%$ & 11 & $8 \%$ \\
\hline $65-74 y$ & 26 & $50 \%$ & 27 & $44 \%$ & 3 & $18 \%$ & 56 & $43 \%$ \\
\hline $75-84 y$ & 23 & $44 \%$ & 27 & $44 \%$ & 10 & $59 \%$ & 60 & $46 \%$ \\
\hline $85+y$ & 0 & $0 \%$ & 1 & $2 \%$ & 2 & $12 \%$ & 3 & $2 \%$ \\
\hline Mean (SD) & $73.9(5.9)$ & & $73.4(6.3)$ & & $75.1(9.4)$ & & $73.8(6.6)$ & \\
\hline \multicolumn{9}{|l|}{ MMSE score } \\
\hline Mean $(S D)$ & $25.1(2.2)$ & & $20.9(3.3)$ & & $14.0(5.4)$ & & $21.7(4.9)$ & \\
\hline \multicolumn{9}{|l|}{ Education (y) } \\
\hline Mean (SD) & $9.8(3.6)$ & & $8.7(3.2)$ & & $8.4(4.2)$ & & $9.1(3.5)$ & \\
\hline \multicolumn{9}{|l|}{ Comorbidity } \\
\hline hypertension & 26 & $50 \%$ & 26 & $43 \%$ & 8 & $47 \%$ & 60 & $46 \%$ \\
\hline diabetes mellitus & 7 & $13 \%$ & 6 & $10 \%$ & 3 & $18 \%$ & 16 & $12 \%$ \\
\hline cardiopathy & 2 & $4 \%$ & 2 & $3 \%$ & 0 & $0 \%$ & 4 & $3 \%$ \\
\hline COPD & 1 & $2 \%$ & 4 & $7 \%$ & 2 & $12 \%$ & 7 & $5 \%$ \\
\hline thyroid disease & 2 & $4 \%$ & 5 & $8 \%$ & 2 & $12 \%$ & 9 & $7 \%$ \\
\hline CKD & 2 & $4 \%$ & 2 & $3 \%$ & 2 & $12 \%$ & 6 & $5 \%$ \\
\hline \multicolumn{9}{|l|}{ Events during study period } \\
\hline COVID-symptoms (patient) & 0 & $0 \%$ & 4 & $7 \%$ & 2 & $12 \%$ & 6 & $5 \%$ \\
\hline COVID-symptoms (relative) & 3 & $6 \%$ & 8 & $13 \%$ & 1 & $6 \%$ & 12 & $9 \%$ \\
\hline Adverse Events & 7 & $13 \%$ & 14 & $23 \%$ & 5 & $29 \%$ & 26 & $20 \%$ \\
\hline Medication change & 11 & $21 \%$ & 21 & $34 \%$ & 5 & $29 \%$ & 37 & $28 \%$ \\
\hline Mean Missed doses (SD) & $2.1(1.8)$ & & $1.6(1.5)$ & & $0.6(1.0)$ & & $1.7(1.6)$ & \\
\hline On-site visits (by June 5th) & 34 & $65 \%$ & 38 & $62 \%$ & 11 & $65 \%$ & 83 & $64 \%$ \\
\hline RT-PCR+ & 0 & $0 \%$ & 0 & $0 \%$ & 0 & $0 \%$ & 0 & $0 \%$ \\
\hline Serology+ & 1 & $3 \%$ & 1 & $3 \%$ & 0 & $0 \%$ & 2 & $2.4 \%$ \\
\hline
\end{tabular}

CDR, Clinical Dementia Rating; SD, standard deviation; COPD, chronic obstructive pulmonary disease; CKD, chronic kidney disease; COVID, Coronavirus Disease.

Following the suspension of on-site visits, clinical trial participants received weekly calls in which overall health status was ascertained using a short questionnaire (see Supplementary Material 1). We were able to perform some of the per protocol assessments remotely (see Supplementary Material 2). A total of 1,018 calls were performed from March 16 to June 5, 2020 (Fig. 1). Only 6 patients (5\%) reported having COVID-19 compatible symptoms (such as cough, fever, etc.) during these calls. Additionally, 12 patients $(9 \%)$ reported to have a close relative (potential contact) with COVID-19 symptoms. The calls also led to ascertaining adverse events in 26 participants (20\%), and changes of concomitant medication in 37 patients (28\%). Beginning March 30, 2020 , study medication started being delivered via a 
160

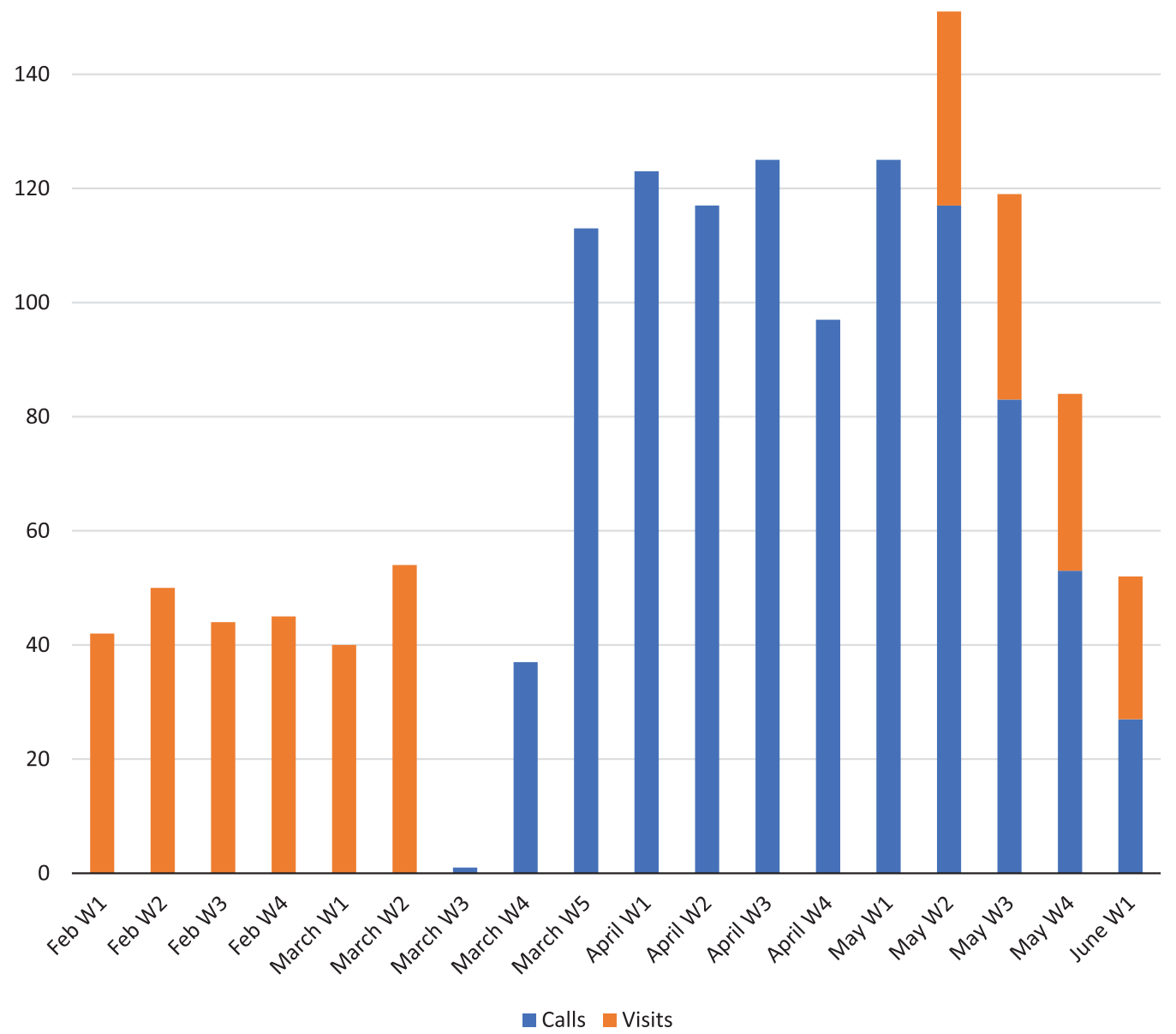

Fig. 1. Weekly calls and on-site visits immediately before and during the study period.

specialized courier service to all participants in trials involving oral administration.

On May 11, 2020, on-site visits began for a group of patients. By June 5th, almost two-thirds of all patients had at least one face-to-face followup visit $(n=83,64 \%)$. Patients from clinical trials with parenteral administration of the investigational product, and those without recent safety MRIs were prioritized. Thus, the proportion of patients with faceto-face visits was higher among randomized patients ( $n=77,71 \%)$, particularly among those receiving an IV-administered study drug $(n=49,85 \%)$. Prior to these visits, diagnostic RT-PCR tests for SARS$\mathrm{CoV}-2$ were performed on patients and on the team of professionals involved in these visits (a total of 13 including administrative personal, neuropsychologists, neurologists, study coordinators, and nurses). All these tests yielded a negative result. Additionally, serological tests for SARS-CoV-2-specific IgG were performed. Two patients and one sub-investigator presented antibodies in these tests $(2.4 \%$ and $7.7 \%$, respectively). These proportions were not statistically different $(p=0.12 ; p=0.28)$ from estimates reported for the province of Barcelona ( $7.1 \%$ in a populationbased sample of 3,693 individuals). Four patients referring COVID-19 symptoms during weekly calls reinitiated on-site visits. RT-PCR and serological tests in all four of them yielded negative results. Seven patients reporting being exposed to a relative with COVID-19 symptoms underwent serological testing and only one of them presented a positive serological result.

All patients and caregivers, as well as personnel complied with the use of PPE, and provided positive feedback on the new safety protocol in place. However, the implementation of PPE did increase 
time demands. We also found that some patients with dementia do not use their masks properly or tend to remove them from their face. These problems could be solved by providing detailed instructions to patients and caregivers as well as frequent reminders of the importance of not removing the masks at any time during study visits.

At the time this manuscript was drafted, Fundació ACE had recorded one drop-out among all 108 randomized patients being followed. Furthermore, this drop-out was unrelated to COVID-19, but due to an adverse event probably related to the study medication. Overall, the mean number of missed doses per patient was $1.7(\mathrm{SD}=1.6)$. While participants enrolled in clinical trials involving oral administration did not missed any dose, the mean number of missed doses per patient among those that required IV or subcutaneous administration of IP was 1.9 $(\mathrm{SD}=1.8)$ and $2.2(\mathrm{SD}=1.4)$, respectively. Study enrollment remains suspended, but all 22 patients who were undergoing screening when this crisis started, are ready to be randomized whenever this becomes possible.

\section{DISCUSSION}

In this paper, we describe a plan aimed at minimizing the impact of the COVID-19 pandemic on active clinical trials in patients with AD. Following this plan, we managed to secure the retention of participants in the clinical trials preventing possible drop-outs. In fact, during these three rather tumultuous months, we have continued all ongoing trials and only recorded a single patient drop-out out of 108 randomized patients. Furthermore, we managed to do so without compromising the safety of clinical trial participants, their families, and our staff. Our plan was only possible thanks to the commitment of Fundació ACE professionals, who made more than one thousand calls to patients during these months while being confined at home with their families. Patients and families are heavily committed to these complex trials, and we found that these regular calls provided a good avenue of communication between patients and the clinic that enabled early detection of any health or even social adverse events occurring to trial participants and helped preserve the trust of patients and families in our institution and its unique model of care. They also prove that is possible to follow up patients with less on-site visits. As the curve of the outbreak flattened, mobility restriction measures started to be gradually relaxed in Spain. The location of Fundació ACE, outside a hospital environment, facilitated resuming in-person visits as early as May 11, 2020. Diagnostic SARS-CoV-2 RTPCR testing performed on all patients prior to these visits confirmed that our strategy was successful in preserving their health without discontinuing any trial. None of the 83 patients and 13 clinical trial personnel evaluated had a positive test result, and only 2 of them and $1 \mathrm{sub}$-investigator showed antibodies for SARS-CoV-2 in the serological tests. These numbers suggest a prevalence of the infection in these populations not higher than that reported for the province of Barcelona, the second largest focus of the outbreak in Spain [17]. We are slowly catching up with all scheduled visits. However, the pace is slower than desired, due to the large number of accumulated visits, the limited availability of staff (now divided in teams/shifts) and additional workload required related to COVID19. This includes SARS-CoV-2 RT-PCR testing on clinic personnel and patients, that is critical for the safety of the trials, and their scientific integrity.

We are all aware of the importance of clinical research in improving health care. This is particularly crucial, in highly prevalent debilitating chronic diseases, such as AD, that still lack effective treatment. We cannot afford to discontinue these trials, nor to threaten the validity of the evidence they provide in these situations [18]. Our group, like others, has been able to come up with ad-hoc plans to cope with the situation and maintain clinical research [19], but not without great burden. Although the overall results of these ad-hoc plans have been positive, we should not have to rely on this type of site-based initiatives. Here we share our experience with the research community to start the conversation of crisis-proof clinical trials protocols because from now on these protocols should include guidelines that clearly define how we should act in this type of catastrophic situations. Furthermore, when possible, clinical trials assessments should be designed in a way they can easily be performed using telemedicine and some safety procedures could be performed using remote monitoring devices. Additionally, optimal solutions should be explored for safe off-site parenteral administration of study drugs. Regular contacts (via phone or videoconference calls) with patients and families beyond scheduled visits should also be considered in these situations, as they are key to improve patient engagement. Some interesting initiatives on this direction are already in place. A recent EU funded call is specifically focused on the new generation digital technolo- 
gies for clinical trials at home [20]. In the case of AD, there is a research project devoted to exploring how mobile technologies (such as smart phones, wearables, and home-based sensors) can be used to assess disease progression and early detection of AD [21]. On another hand, the inclusion of diagnostic testing for the infectious disease causing the pandemic could be implemented as a safety assessment for the participants of clinical trials, especially if they are immunological therapies in at-risk populations. Finally, in our experience, fluent communication with clinical trial sponsors was key to successfully managing the situation. We propose the creation of ad-hoc crisis committees where representatives from the sponsor, the clinical investigators and the regulatory authorities can discuss and make timely decisions aimed at preserving the safety of all participants without compromising the validity of the results of the trial.

In conclusion, our experience suggests that the disruptive effects of public health crisis of this magnitude in clinical research can be minimized using strategic planning. These plans should not rely on individual initiatives but should be harmonized across study sites and routinely included in study protocols. As new digital tools are developed and patient remote assessment becomes a standard, clinical trials should evolve to become more robust to this type of crisis, and incidentally, more accessible to populations who live far from main research centers. We should seize the opportunity to improve the way we do clinical research in dementia patients. We owe it to the victims of this terrible pandemic.

\section{ACKNOWLEDGMENTS}

No funding was received for this work. This work would not have been possible without the assistance of Fundació ACE administrative staff including Carmen Chaler, Idoia Ruiz, Marta Lozano, Salvador Almagro, $\mathrm{M}^{\mathrm{a}}$ Jesús Allué, Verónica Ferrer, Angela Rodríguez, Rafael Barba, Eva Canela, Charo Romero, Mercè Roca, Esther Pelejà, $\mathrm{M}^{\mathrm{a}}$ José Castillón, Cristina Arso, and Purificación Conde.

Authors' disclosures available online (https:// www.j-alz.com/manuscript-disclosures/20-0750r2).

\section{SUPPLEMENTARY MATERIAL}

The supplementary material is available in the electronic version of this article: https://dx.doi.org/10. 3233/JAD-200750.

\section{REFERENCES}

[1] Guan WJ, Ni ZY, Hu Y, Liang WH, Ou CQ, He JX, Liu L, Shan H, Lei CL, Hui DSC, Du B, Li LJ, Zeng G, Yuen KY, Chen RC, Tang CL, Wang T, Chen PY, Xiang J, Li SY, Wang JL, Liang ZJ, Peng YX, Wei L, Liu Y, Hu YH, Peng P, Wang JM, Liu JY, Chen Z, Li G, Zheng ZJ, Qiu SQ, Luo J, Ye CJ, Zhu SY, Zhong NS (2020) Clinical characteristics of coronavirus disease 2019 in China. N Engl J Med 382, $1708-1720$.

[2] World Health Organization (2020) WHO DirectorGeneral's opening remarks at the media briefing on COVID-19 - 11 March 2020. https://www.who.int/dg/ speeches/detail/who-director-general-s-opening-remarksat-the-media-briefing-on-covid-19-11-march-2020

[3] FDA (2020) FDA Guidance on Conduct of Clinical Trials of Medical Products during COVID-19 Pandemic Guidance for Industry, Investigators, and Institutional Review Boards. https://www.fda.gov/regulatory-information/search-fdaguidance-documents/fda-guidance-conduct-clinicaltrials-medical-products-during-covid-19-public-healthemergency

[4] European Medicines Agency (EMA) (2020) Guidance on the Management of Clinical Trials during the COVID-19 (Coronavirus) pandemic. https://ec.europa.eu/health/sites/ health/files/files/eudralex/vol-10/guidanceclinicaltrials_ covid19_en.pdf

[5] Agencia Española de Medicamentos y Productos Sanitarios (AEMPS) (2020) Medidas excepcionales aplicables a los ensayos clínicos para gestionar los problemas derivados de la emergencia por COVID-19. https://www.aemps.gob.es/ informa/notasinformativas/medicamentosusohumano-3/ 2020-medicamentosusohumano-3/medidas-excepcionalesaplicables-a-los-ensayos-clinicos-para-gestionar-losproblemas-derivados-de-la-emergencia-por-covid$19 /$ ?lang=en

[6] Ousset PJ, Vellas B (2020) Impact of the Covid-19 outbreak on the clinical and research activities of memory clinics: An Alzheimer's disease center facing the Covid-19 crisis. J Prev Alzheimers Dis 7, 197-198.

[7] Geerts H, van der Graaf PH (2020) Salvaging CNS clinical trials halted due to COVID-19. CPT Pharmacometrics Syst Pharmacol 9, 367-370.

[8] Weinberg MS, Patrick RE, Schwab NA, Owoyemi P, May R, McManus AJ, Gerber J, Harper DG, Arnold SE, Forester B (2020) Clinical trials and tribulations in the COVID-19 era. Am J Geriatr Psychiatry 28, 913-920.

[9] Singh AG, Chaturvedi P (2020) Clinical trials during COVID-19. Head Neck 42, 1516-1518.

[10] Wang H, Wu J, Wei Y, Zhu Y, Ye D (2020) Surgical volume, safety, drug administration, and clinical trials during COVID-19: Single-center experience in Shanghai, China. Eur Urol 78, 120-122.

[11] United Nations (2020) Policy Brief: The Impact of COVID19 on older persons. http://www.un.org/development/desa/ ageing/wp-content/uploads/sites/24/2020/05/COVIDOlder-persons.pdf

[12] Journal T (2020) Alzheimer's disease research enterprise in the era of COVID-19/SARS-CoV-2. Alzheimers Dement 16, 587-588.

[13] Brown EE, Kumar S, Rajji TK, Pollock BG, Mulsant BH (2020) Anticipating and mitigating the impact of the COVID-19 pandemic on Alzheimer's disease and related dementias. Am J Geriatr Psychiatry 28, 712-721. 
[14] Boada M, Tárraga L, Hernández I, Valero S, Alegret M, Ruiz A, Lopez OL, Becker JT (2014) Design of a comprehensive Alzheimer's disease clinic and research center in Spain to meet critical patient and family needs. Alzheimers Dement 10, 409-415.

[15] Benaque A, Gurruchaga MJ, Abdelnour C, Hernández I, Cañabate P, Alegret M, Rodríguez I, Rosende-Roca M, Tartari JP, Esteban E, López R, Gil S, Vargas L, Mauleón A, Espinosa A, Ortega G, Sanabria A, Pérez A, Alarcón E, González-Pérez A, Marquié M, Valero S, Tárraga L, Ruiz A, Boada M, for the Research Center and Memory Clinic FACE (2020) Dementia care in times of COVID-19: Experience at Fundació ACE in Barcelona, Spain. J Alzheimers Dis 76, 33-40.

[16] Insituto de Salud Carlos III (2020) Estudio ene-COVID19: Primera ronda estudio nacional de sero-epidemiología de la infección por SARS-COV-2 en España Mapa provincial de Anticuerpos IgG anti SARS-CoV2.
[17] Covid-19: Spain's macro study shows just 5.2\% of population has contracted the coronavirus | Society | EL PAÍS in English, https://english.elpais.com/society/2020-06-05/ spains-macro-study-show-just-52-of-population-hascontracted-the-coronavirus.html

[18] McDermott MM, Newman AB (2020) Preserving clinical trial integrity during the coronavirus pandemic. JAMA 323, 2135-2136.

[19] Takeda C, Guyonnet S, Ousset PJ, Soto M, Vellas B (2020) Toulouse Alzheimer's Clinical Research Center recovery after the COVID-19 crisis: Telemedicine an innovative solution for clinical research during the coronavirus pandemic. J Prev Alzheimers Dis 7, 301-304.

[20] Calls 2020 | ECSEL Joint Undertaking, https://www.ecsel. $\mathrm{eu} / \mathrm{calls} / \mathrm{calls}-2020$

[21] Welcome to RADAR-AD | Radar-AD, https://www.radarad.org/ 